\title{
Wave Interactions for Chaplygin Gas with Combustion
}

\section{Yujin Liu}

School of Mathematics and Statistics, Shandong University of Technology, Zibo, China

Email: yjliu98@126.com

How to cite this paper: Liu, Y.J. (2021) Wave Interactions for Chaplygin Gas with Combustion. Journal of Applied Mathematics and Physics, 9, 683-693.

https://doi.org/10.4236/jamp.2021.94049

Received: March 17, 2021

Accepted: April 18, 2021

Published: April 21, 2021

Copyright $\odot 2021$ by author(s) and Scientific Research Publishing Inc. This work is licensed under the Creative Commons Attribution International License (CC BY 4.0).

http://creativecommons.org/licenses/by/4.0/

\begin{abstract}
The elementary wave interactions for the Chapman-Jouguet model with combustion are investigated. We obtain the unique solution of the initial value problem under the global entropy conditions. We analyze the elementary wave interactions in the phase plane and construct uniquely the solution of this initial value problem. It is found that the combustion wave solution of the corresponding Riemann may be extinguished after perturbation which shows that the unburnt gas is unstable.
\end{abstract}

\section{Keywords}

Riemann Problem, Chaplygin Gas, Detonation Wave, Deflagration Wave

\section{Introduction}

In this paper, we study the following equations

$$
\left\{\begin{array}{l}
\rho_{t}+(\rho u)_{x}=0, \\
(\rho u)_{t}+\left(\rho u^{2}+p\right)_{x}=0, \\
(\rho E)_{t}+(\rho u E+p u)_{x}=0, \\
q(x, t)= \begin{cases}0 & \text { if } \sup _{0 \leq y \leq t} T(x, y)>T_{i} \\
q(x, 0) & \text { otherwise, }\end{cases}
\end{array}\right.
$$

where $\rho, u, p<0, T$ are the density, velocity, pressure, temperature respectively. $T_{i}$ is the ignition temperature and $q$ is the chemical binding energy. The total energy $E=\frac{u^{2}}{2}+e+q$, where $e$ is the internal energy. The state equation is given by $p=-\frac{1}{\rho}$ and $e=-\frac{p}{2 \rho}$. The process of combustion is exothermic [1]. The early study for Chaplygin gas can be found in [2] [3]. 
In [4], the authors investigated the two-dimensional Riemann problem for isentropic Chaplygin gas dynamic system according to different combinations of four elementary waves and they delivered a complete classification to the above problem.

In [5], the authors studied two new types of self-similar solutions to the Chaplygin gas model in two space dimensions and they constructed the solutions to the interaction of two rarefaction simple waves.

The authors [6] investigated several two-dimensional Riemann problems for Chaplygin gas model and proved the existence of the unique solution.

In [7], the authors studied the appearance of delta shock wave and vacuum state in the vanishing pressure limit of Riemann solutions to the non-isentropic generalized Chaplygin gas equations.

The authors [8] constructed the solutions of Riemann problem for the isentropic relativistic Euler equations with the extended Chaplygin gas and studied the asymptotic limits of solutions to the Riemann problem for the relativistic Euler equations.

We use usually two mathematical models to investigate the combustion phenomena. One is the Chapman-Jouguet (CJ) model, and the other is the Zeldovich-von Neumann-Döring (ZND) model [1] [9]. In [1], the authors obtained the partial combustion solutions for the CJ model. In [10], the authors investigated the following $\mathrm{CJ}$ model

$$
\left\{\begin{array}{l}
u_{t}+p_{x}=0, \\
\tau_{t}-u_{x}=0, \\
E_{t}+(u p)_{x}=0, \\
q(x, t)= \begin{cases}0 & \text { if } \sup _{0 \leq y \leq t} T(x, y)>T_{i} \\
q(x, 0) & \text { otherwise, }\end{cases}
\end{array}\right.
$$

here $\tau=\frac{1}{\rho}$ and $\tau$ is the specific volume, $p>0$. It is followed that there is unique solution for (2). In [11] we got uniquely the solution of the generalized Riemann problem for (2).

In [12] the authors studied the following selfsimilar ZND model

$$
\left\{\begin{array}{l}
u_{t}+p_{x}=0, \\
\tau_{t}-u_{x}=0, \\
E_{t}+(u p)_{x}=0, \\
q(x, t)=-\frac{k}{t} \varphi(T) q .
\end{array}\right.
$$

and constructed its unique solution under some hypothesis. In [13], the authors determined when the temperatures along the burning solutions of (3) are higher than the ignition temperature which is the necessary condition to study the limit behavior as the reaction velocity tends to infinity.

In [14], we obtained the Riemann solution of (1) with the following initial 
values

$$
(\tau, u, p, q)= \begin{cases}\left(\tau^{-}, u^{-}, p^{-}, q^{-}\right) & \text {when } x<0, \\ \left(\tau^{+}, u^{+}, p^{+}, q^{+}\right) & \text {when } x>0 .\end{cases}
$$

In this paper, we investigate the initial value problem for (1) with the following initial values

$$
(\tau, u, p)(x, 0)= \begin{cases}\left(\tau^{l}, u^{l}, p^{l}\right), & -\infty<x \leq x_{1}, \\ \left(\tau^{m}, u^{m}, p^{m}\right), & x_{1}<x \leq x_{2}, \\ \left(\tau^{r}, u^{r}, p^{r}\right), & x_{2}<x<\infty .\end{cases}
$$

In [15], we considered the generalized Riemann problem for (1) and found some interesting phenomena.

It is shown that the structures of the Riemann solutions can retain their forms after perturbation for most of the cases, while for some other cases, the perturbation can make a big difference. It is observed that although there is no combustion wave in the corresponding Riemann solution, the combustion wave may occur after perturbation. It shows the instability of the unburnt gas.

The paper is arranged as follows. In Section 2, we list the preliminaries. We obtain the unique solution for the initial value problem (1) and (5) in Section 3. Section 4 gives our main result.

\section{Preliminaries}

In this section we give some preliminaries [14] [15] [16] [17].

The characteristic roots for (1) are given by

$$
\mu_{1,3}=u \pm \sqrt{\frac{-p}{\rho}}, \mu_{2}=u,
$$

it follows that (1.1) is strictly hyperbolic. The right characteristic vector of $\lambda_{i}(i=1,2,3)$ is

$$
\vec{r}_{1,3}=\left(1, \pm \frac{1}{\rho} \sqrt{\frac{-p}{\rho}}, \frac{-p}{\rho}\right)^{\mathrm{T}}, \vec{r}_{2}=(1,0,0)^{\mathrm{T}} .
$$

From $\nabla \lambda_{i} \cdot \vec{r}_{i} \equiv 0, i=1,2,3$, all characteristic fields are linearly degenerate.

The rarefaction wave curves $\vec{R}(l)$ (or $\bar{R}(l))$ are

$$
\left\{\begin{array}{l}
p \rho=p_{l} \rho_{l}, \\
u=u_{l} \pm \frac{p-p_{l}}{\sqrt{-p_{l} \rho_{l}}}, \quad\left(p>p_{l}, \text { or } p<p_{l}\right),
\end{array}\right.
$$

and the shock wave curves $\vec{S}(l) \quad$ (or $\bar{S}(l))$ are

$$
\left\{\begin{array}{l}
p \rho=p_{l} \rho_{l}, \\
\frac{u-u_{l}}{p-p_{l}}= \pm \sqrt{-\frac{1}{p_{l} \rho_{l}}}, \quad\left(p_{l}>p, \text { or } p_{l}<p\right) .
\end{array}\right.
$$

The contact discontinuity $J$ is 


$$
\left\{\begin{array}{l}
{[u]=[p]=0} \\
\rho_{l} \neq \rho_{r}
\end{array}\right.
$$

Suppose

$$
\begin{aligned}
& \rho=\rho_{0}+\omega(t) \delta(x-x(t)), \rho_{0}= \begin{cases}\rho_{l}, & x<x(t), \\
\rho_{r}, & x>x(t),\end{cases} \\
& u(x, t)= \begin{cases}u_{l}, & x<x(t), \\
u_{\delta}, & x=x(t), \\
u_{r}, & x>x(t),\end{cases} \\
& p(x, t)= \begin{cases}p_{l}, & x<x(t), \\
0, & x=x(t), \\
p_{r}, & x>x(t) .\end{cases}
\end{aligned}
$$

When $\rho_{r} \neq \rho_{l}$,

$$
\begin{gathered}
\omega(t)=\sqrt{\rho_{l} \rho_{r}\left(u_{r}-u_{l}\right)^{2}-\left(\rho_{r}-\rho_{l}\right)\left(p_{r}-p_{l}\right) t} \\
u_{\delta}=\frac{\rho_{r} u_{r}-\rho_{l} u_{l}+\frac{\mathrm{d} \omega(t)}{\mathrm{d} t}}{\rho_{r}-\rho_{l}},
\end{gathered}
$$

when $\rho_{r}=\rho_{l}$,

$$
\begin{gathered}
\omega(t)=\left(\rho_{l} u_{l}-\rho_{r} u_{r}\right) t \\
u_{\delta}=\frac{1}{2}\left(u_{r}+u_{l}\right) .
\end{gathered}
$$

Further, the delta shock $S_{\delta}$ satisfies the entropy condition

$$
u_{r}+\sqrt{-\frac{p_{r}}{\rho_{r}}}<\frac{\mathrm{d} x(t)}{\mathrm{d} t}<u_{l}-\sqrt{-\frac{p_{l}}{\rho_{l}}} .
$$

The non-combustion wave curves can be shown in the phase plane $(u, p)$ (Figure 1).

From the R-H condition

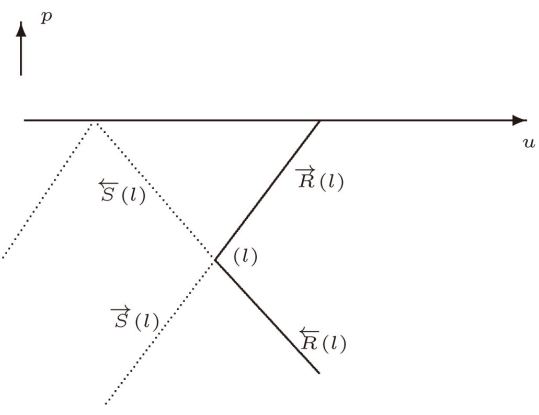

(i)
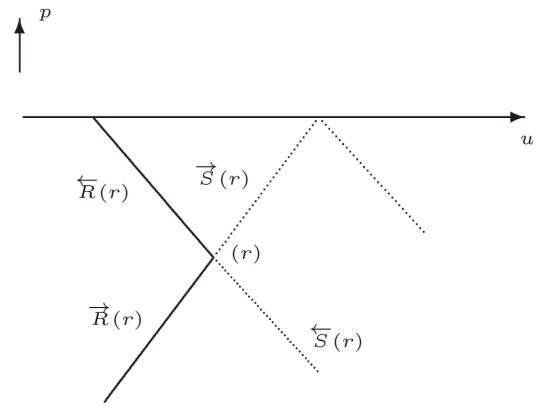

(ii)

Figure 1. The non-combustion wave curves. (i) The backward wave curves; (ii) The forward wave curves. 


$$
\left\{\begin{array}{l}
\eta[u]=[p], \\
\eta[\tau]=-[u], \\
\eta[E]=[u p],
\end{array}\right.
$$

we find that

$$
-\tau_{r} p+p_{r} \tau=2 q_{0}>0 .
$$

The forward combustion wave curve in $(u, p)$ (Figure 2) is

$$
\vec{D}(r): \frac{u-u_{r}}{p-p_{r}}=\sqrt{-\frac{2 q_{0}+\tau_{r}\left(p-p_{r}\right)}{p_{r}\left(p-p_{r}\right)}},
$$

where $p_{r}<p<0$ or $p<p_{r}-\frac{2 q_{0}}{\tau}$. When $p_{r}<p<0$, it is the detonation wave curve and when $p<p_{r}-2 q_{0}^{\tau_{r}} \rho_{r}$, it is the deflagration wave curve [1].

When $q^{+}>0$, the forward wave curve $\vec{W}(+)$ is

$$
\vec{W}(+) \doteq \vec{W}_{S}(+) \bigcup S_{\delta}(+) \cup \overrightarrow{D F}(+) \cup \overrightarrow{D T}(+),
$$

here

$$
\vec{W}_{S}(+) \doteq \vec{R}(+) \bigcup \vec{S}(+)
$$

When $q^{+}=0, q^{-}=0$, it is studied in [16]. Otherwise, we proceed as follows.

Case 2.1. When $q^{-}=0, q^{+}=q_{0}>0$.

The backward wave curve is $\bar{W}(-)=\bar{W}_{S}(-) \cup S_{\delta}(-)$, and the forward wave curve is $\vec{W}(+)=\vec{W}_{S}(+) \cup S_{\delta}(+) \cup \overrightarrow{D F}(+) \cup \overrightarrow{D T}(+)$ (Figure 3).

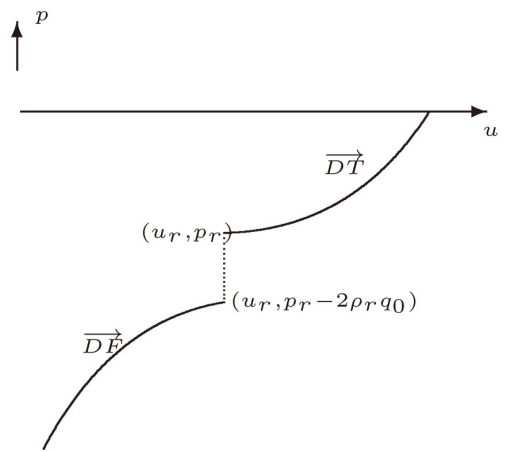

Figure 2. The combustion wave curve in $(u, p)$.

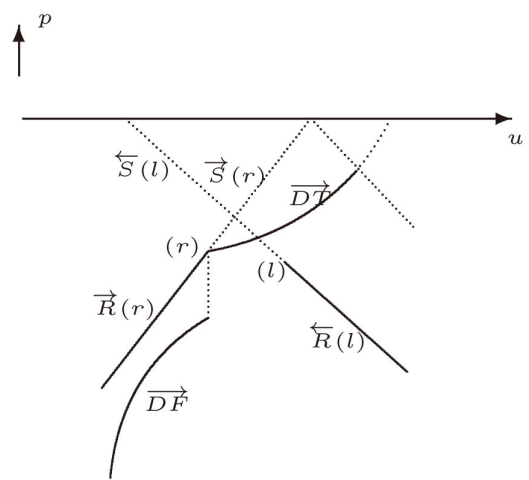

Figure 3. The wave curves in Case 2.1. 
Subcase 2.1.1 $u_{-}-u_{+}<\sqrt{-\frac{p_{-}}{\rho_{-}}}+\sqrt{-\frac{p_{+}}{\rho_{+}}}$.

To guarantee the unique solution of the Riemann problem, we give the global entropy conditions:

We select the unique solution from the four intersection points (at most) in the following order:

1) the solution with the parameter $\delta$ as small as possible, where $\delta$ is defined as oscillation frequency of $T(\zeta)$ between the set $\left\{\zeta \in R^{1}: T(\zeta) \leq T_{i}\right\}$ and the set $\left\{\zeta \in R^{1}: T(\zeta)>T_{i}\right\}$;

$2)$ the solution containing the combustion wave as many as possible.

For simplicity, $*_{1}$ is the intersection point of $\overleftarrow{W}_{S}(-)$ and $\vec{W}_{S}(+)$ and $*_{2}$ is the intersection point of $\bar{W}_{S}(-)$ and $\overrightarrow{D F}(+)$ or $\overrightarrow{D F}(+)$ The temperature is respectively $T_{1}, T_{2}$ at the point $*_{1}, *_{2}$ (Figure 4 ).

1) When $T_{-}>T_{i}, T_{2}>T_{i}$, then $\delta\left(*_{1}\right)=1, \delta\left(*_{2}\right)=1$, due to (ii), we pick out $*_{2}$ and get the combustion wave solution $D F$ or $D T$ (Figure 4(i)).

2) When $T_{-}>T_{i}, T_{2} \leq T_{i}\left(\Rightarrow T_{1} \leq T_{i}\right)$, then $\delta\left(*_{1}\right)=1, \delta\left(*_{2}\right)=3$, due to $(i)$, we pick out $*_{1}$ and get the noncombustion wave solution (Figure 4(ii)).

3) When $T_{-} \leq T_{i}, T_{1} \leq T_{i}$, then $\delta\left(*_{1}\right)=0, \delta\left(*_{2}\right)=2$, due to (i), we pick out $*_{1}$ and get the noncombustion wave solution (Figure 4(ii)).

4) When $T_{-} \leq T_{i}, T_{1}>T_{i}\left(\Rightarrow T_{2}>T_{i}\right)$, then $\delta\left(*_{1}\right)=2, \delta\left(*_{2}\right)=2$, due to (ii), we pick out $*_{2}$ and get the combustion wave solution DF or DT (Figure 4(i)).

Subcase 2.1.2 $u_{-}-u_{+} \geq \sqrt{-\frac{p_{-}}{\rho_{-}}}+\sqrt{-\frac{p_{+}}{\rho_{+}}}$.

It is shown that we can get the delta shock wave solution $S_{\delta}$ [14] [15] [16].

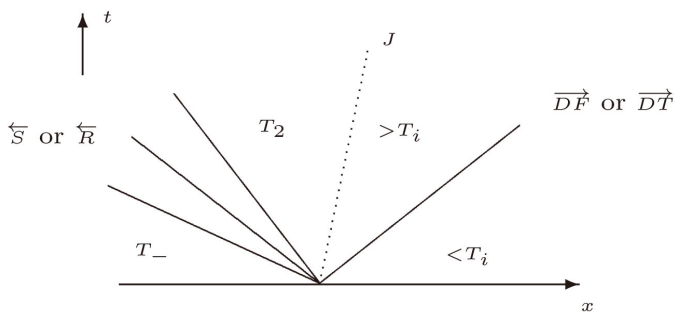

(i)

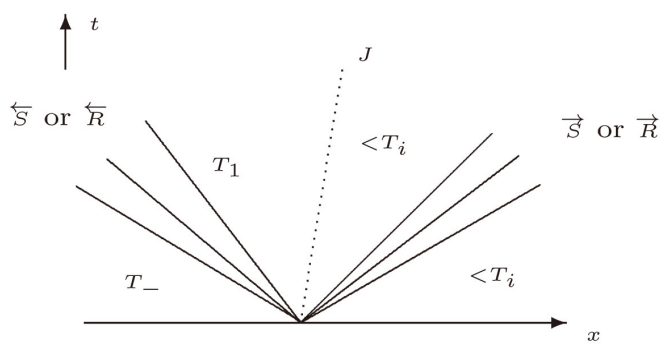

(ii)

Figure 4. The analysis in Subcase 2.1.1. (i) combustion wave solution; (ii) non-combustion wave solution. 
Case 2.2. When $q^{-}>0, q^{+}>0$. We go on as follows.

We know that $\bar{W}(-)=\bar{W}_{S}(-) \cup S_{\delta}(-) \cup \overleftarrow{D T}(-) \cup \overline{D F}(-)$, and $\vec{W}(+)=\vec{W}_{S}(+) \cup S_{\delta}(+) \cup \overrightarrow{D T}(+) \cup \overrightarrow{D F}(+)$ (Figure 5).

Subcase 2.2.1 When $u_{-}-u_{+}<\sqrt{-\frac{p_{-}}{\rho_{-}}}+\sqrt{-\frac{p_{+}}{\rho_{+}}}$(Figure 6).

Since we have $\delta=0$ for Case (1), $\delta=2$ for Case (2)-(4), we pick out the intersection point a and get the non-combustion wave solution.

Subcase 2.2.2 When $u_{-}-u_{+} \geq \sqrt{-\frac{p_{-}}{\rho_{-}}}+\sqrt{-\frac{p_{+}}{\rho_{+}}}$. We get the delta shock wave solution for this case similarly.

Theorem 2.1. Under the given global entropy conditions, we obtain uniquely the Riemann solution for (1) and (4).

\section{Elementary Wave Interactions for (1) with (5)}

For simplicity, we just consider the case that the delta shock wave does not appear. We consider the four kinds of wave interactions:

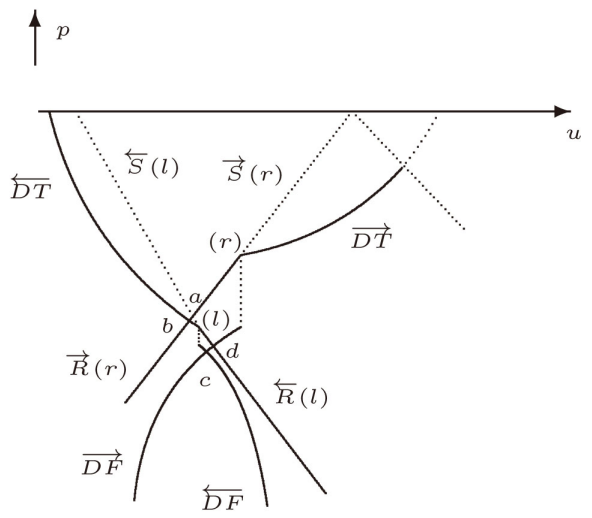

Figure 5. The wave curves in Case 2.2.

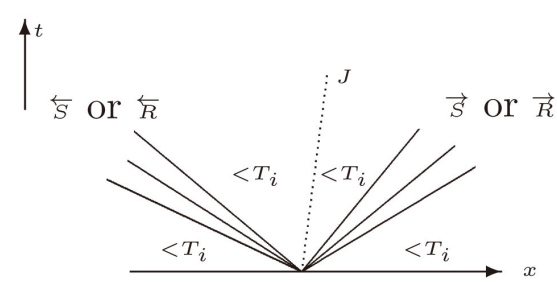

(i)

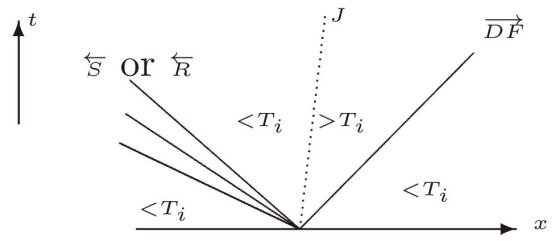

(iii)

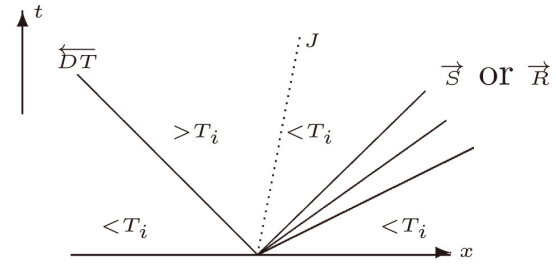

(ii)

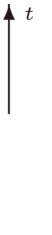

(iv)

Figure 6. The discussions in Subcase 2.2.1. Case (i) solution of the point a; Case (ii) solution of the point b; Case (iii) solution of the point c; Case (iv) solution of the point $d$. 
A combustion wave $\overline{D T}$ and a shock wave $\bar{S}$, a combustion wave $\overline{D F}$ and a shock wave $\bar{S}$, a combustion wave $\overline{D T}$ and a rarefaction wave $\bar{R}$, a combustion wave $\overline{D F}$ and a rarefaction wave $\bar{R}$.

Case 3.1. $\overline{D T}$ and $\bar{S}$ (Figure 7)

We know $\mu_{m}<\eta_{1}<\mu_{l}$ for $\overline{D T}$, and $\mu_{r}<\eta_{2}<\mu_{m}$ for $\bar{S}$, where $\eta_{1}$ and $\eta_{2}$ are the velocity of $\overline{D T}$ and $\bar{S}$ respectively. $(m) \in \bar{W}_{D T}(l),(r) \in \bar{S}(m)$. From the global entropy conditions, we know that the solution for this case is

$$
\overleftarrow{D T}+\vec{S} \rightarrow \vec{S}+J+\vec{S} .
$$

Theorem 3.1. For the wave interaction between the shock wave and the detonation wave, we find that it may extinguish the combustion wave which shows that the unburnt gas is unstable. And after the elementary wave interaction process, we observe that the contact discontinuity may emerge.

Case 3.2. $\overleftarrow{D F}$ and $\bar{S}$

In this case (Figure 8), it follows that the shock wave will overtake the combustion wave and we need to investigate the new initial value problem. Considering the fact that $(m) \in \bar{W}_{D F}(l)$, and due to $(r) \in \bar{S}(m)$, we get that the results from the global entropy conditions are described by

$$
\overline{D F}+\bar{S} \rightarrow \bar{R} \text { or } \bar{S}+J+\vec{S}
$$
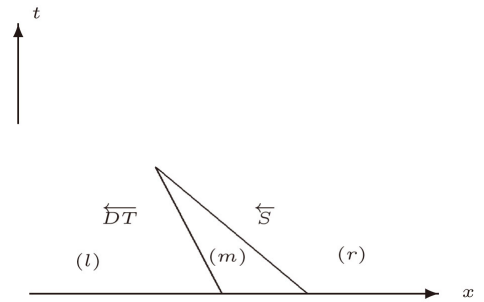

$\uparrow^{p}$

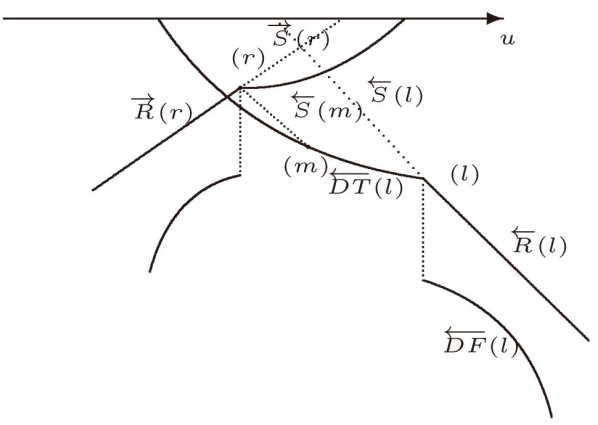

Figure 7. The interaction of $\overline{D T}$ and $\bar{S}$.
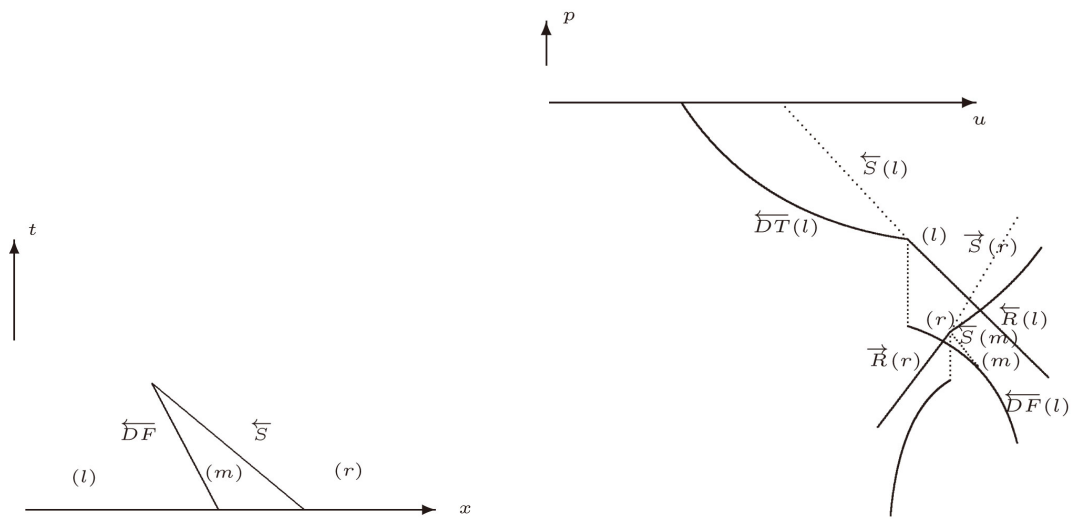

Figure 8. The interaction of $\overline{D F}$ and $\bar{S}$. 
Theorem 3.2. In this situation, the result of the wave interaction between the shock wave and the deflagration wave is that the deflagration combustion wave may be extinguished. Furthermore, we find that the contact discontinuity may emerge after the elementary wave interaction process.

Case 3.3. $\overline{D T}$ and $\bar{R}$

For this situation (Figure 9), it follows that $\overline{D T}$ will interact with $\bar{R}$, and the new initial value problem is formed. Due to $(m) \in \bar{W}_{D T}(l)$, and considering that $(r) \in \bar{R}(m)$, again from the global entropy conditions, we know that the result of the wave interaction process can be given by

$$
\overline{D T}+\bar{R} \rightarrow \bar{S} \text { or } \bar{R}+J+\vec{S} \text {. }
$$

Theorem 3.3. In this situation, the result of the wave interaction between the rarefaction wave and the detonation wave is that it may extinguish the combustion wave. It shows that the unburnt gas is unstable. Further, we find that the contact discontinuity may emerge after the elementary wave interaction process.

Case 3.4. $\overleftarrow{D F}$ and $\bar{R}$

In this case (Figure 10), we know that the rarefaction wave will interact with the combustion wave. Then a new initial value problem is formed. Considering the fact $(m) \in \bar{W}_{D F}(l)$ and $(r) \in \bar{S}(m)$, due to the global entropy conditions we get that the result of the wave interaction can be described by

$$
\overline{D F}+\bar{R} \rightarrow \bar{R}+J+\vec{S} \text {. }
$$
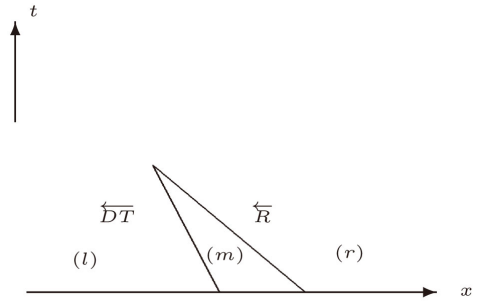

Figure 9. The interaction of $\overline{D T}$ and $\bar{R}$.
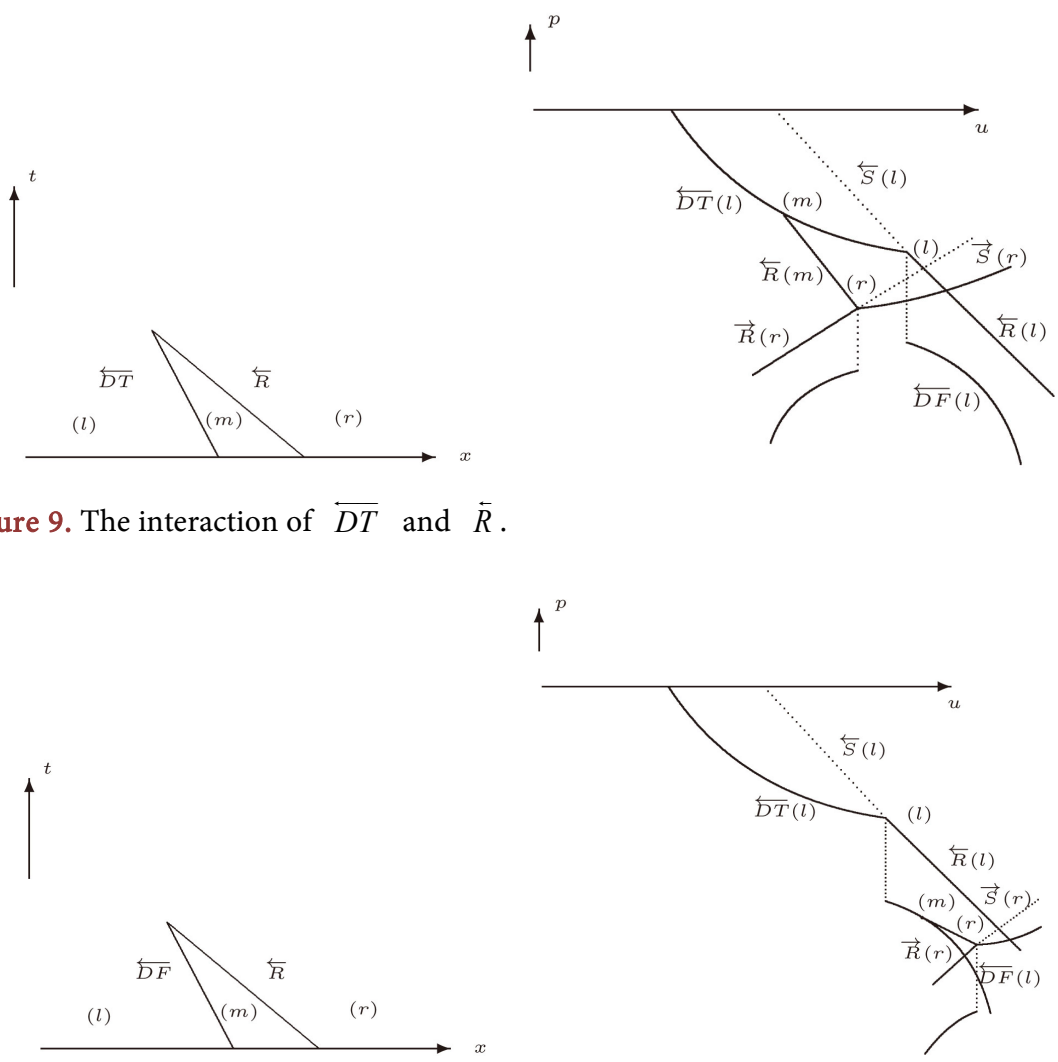

Figure 10. The interaction of $\overline{D F}$ and $\bar{R}$. 
Theorem 3.4. In this situation, the result of the wave interaction between the rarefaction wave and the deflagration wave is the deflagration wave may be extinguished. Furthermore, we find that the contact discontinuity may emerge after the elementary wave interaction process.

\section{Conclusions}

Based on the above analysis, we conclude our main results.

Theorem 4.1. There exists unique solution for the initial value problem (1) and (5). By studying the concrete elementary wave interactions, we observe interesting combustion phenomenon. The combustion process may be extinguished which reveals that the unburnt gas is unstable.

For simplicity, we suppose that the reaction rate in the combustion model (1) is infinite, Of course, it is still an important model to study the combustion phenomena in our many applications in every way. In our further study, we will study the self-similar ZND model which has a finite reaction rate.

\section{Funding}

Supported by the Foundation for Young Scholars of Shandong University of Technology (No. 115024).

\section{Conflicts of Interest}

The author declares no conflicts of interest regarding the publication of this paper.

\section{References}

[1] Courant, R. and Friedrichs, K.O. (1948) Supersonic Flow and Shock Waves. Interscience, New York.

[2] Chaplygin, S. (1904) On Gas Jets. Scientific Memoirs, Moscow University Mathematic Physics, 21, 1-121.

[3] Brenier, Y. (2005) Solutions with Concentration to the Riemann Problem for One-Dimensional Chaplygin Gas Equations. Journal of Mathematical Fluid Mechanics, 7, S326-S331. https://doi.org/10.1007/s00021-005-0162-x

[4] Guo, L.H., Sheng, W.C. and Zhang, T. (2010) The Two-Dimensional Riemann Problem for Isentropic Chaplygin Gas Dynamics System. Communications on Pure \& Applied Analysis, 9, 431-458. https://doi.org/10.3934/cpaa.2010.9.431

[5] Lai, G., Sheng, W.C. and Zheng, Y.X. (2011) Simple Waves and Pressure Delta Waves for a Chaplygin Gas in Two-Dimensions. Discrete \& Continuous Dynamical Systems, 31, 489-523. https://doi.org/10.3934/dcds.2011.31.489

[6] Serre, D. (2009) Multidimensional Shock Interaction for a Chaplygin Gas. Archive for Rational Mechanics and Analysis, 191, 539-577. https://doi.org/10.1007/s00205-008-0110-Z

[7] Ding, Q.X. and Guo, L.H. (2019) The Vanishing Pressure Limit of Riemann Solutions to the Non-Isentropic Euler Equations for Generalized Chaplygin Gas. Advances in Mathematical Physics, 2019, Article ID: 5253717. https://doi.org/10.1155/2019/5253717 
[8] Zhang, Y.F. and Sun, M.N. (2020) Concentration Phenomenon of Riemann Solutions for the Relativistic Euler Equations with the Extended Chaplygin Gas. Acta Applicandae Mathematicae, 170, 539-568. https://doi.org/10.1007/s10440-020-00345-7

[9] Williams, F.A. (1985) Combustion Theory. Benjamin Commings, Menlo Park.

[10] Zhang, T. and Zheng, Y.X. (1989) Riemann Problem for Gasdynamic Combustion. Journal of Differential Equations, 77, 203-230. https://doi.org/10.1016/0022-0396(89)90142-3

[11] Liu, Y.J. and Sheng, W.C. (2011) Generalized Riemann Problem for Gas Dynamic Combustion. Applied Mathematics and Mechanics, 32, Article No. 1079. https://doi.org/10.1007/s10483-011-1482-6

[12] Tan, D.C. and Zhang, T. (1992) Riemann Problem for the Selfsimilar ZND Model in Gas Dynamical Combustion. Journal of Differential Equations, 95, 331-369. https://doi.org/10.1016/0022-0396(92)90035-L

[13] Hsu, C.H. and Lin, S.S. (1997) Some Qualitative Properties of the Riemann Problem in Gas Dynamical Combustion. Journal of Differential Equations, 140, 10-43. https://doi.org/10.1006/jdeq.1997.3304

[14] Liu, Y.J. (2015) Riemann Problem for Chaplygin Gas with Combustion. Appl. Ana., 94, 1877-1887. https://doi.org/10.1080/00036811.2014.956732

[15] Liu, Y.J. (2019) The Generalized Riemann Problem for Chaplygin Gas with Combustion. Journal of Applied Mathematics and Physics, 7, 2738-2750. https://doi.org/10.4236/jamp.2019.711187

[16] Zhu, L. (2010) Chaplygin Gas Dynamic System in Adiabatic Flow. Master Thesis, Shanghai University, Shanghai.

[17] Sheng, W.C. and Zhang, T. (1999) The Riemann Problem for the Transportation Equations in Gas Dynamics. Memoirs of the American Mathematical Society, 137, 1-77. https://doi.org/10.1090/memo/0654 